\title{
Comunicação
}

[Communication]

\section{Estudo retrospectivo ecodopplercardiográfico das principais cardiopatias diagnosticadas em cães}

[Echodopplercardiographic retrospective study of the main cardiopathies diagnosed in dogs]

\author{
M.G. Castro ${ }^{1}$, J.C.C. Veado ${ }^{2}$, E.F. Silva ${ }^{3}$, R.B. Araújo $^{2}$ \\ ${ }^{1}$ Médica veterinária residente - Hospital Veterinário - EV-UFMG - Belo Horizonte, MG \\ ${ }^{2}$ Escola de Veterinária - UFMG - Belo Horizonte, MG \\ ${ }^{3}$ Médico veterinário - Hospital Veterinário - EV-UFMG - Belo Horizonte, MG
}

Entre os diversos métodos de auxílio ao diagnóstico das doenças cardíacas, a ecocardiografia tem sido cada vez mais utilizada na medicina veterinária por ser um método dinâmico e não-invasivo de avaliação do coração (Boon, 1998; Kienle e Thomas, 2005). A ecocardiografia permite, por meio de visualização direta das câmaras cardíacas, uma avaliação da relação espacial entre as estruturas, dos movimentos cardíacos e características dos fluxos sanguíneos, possibilitando o diagnóstico de várias alterações cardíacas, como afecções valvulares, miocardiopatias, anomalias congênitas e doenças pericárdicas (Muzzi, 2002; Kienle e Thomas, 2005).

O conhecimento da prevalência das afecções cardíacas em cães é de fundamental importância para o clínico de pequenos animais, auxiliando na formulação de diagnósticos diferenciais e no estabelecimento de um plano terapêutico adequado. Assim, o objetivo deste trabalho é apresentar a frequência das principais cardiopatias diagnosticadas em cães atendidos pelo serviço de ecocardiografia do Hospital Veterinário (HV) da Universidade Federal de Minas Gerais (UFMG).

Por meio de um estudo retrospectivo, foram avaliados exames ecodopplercardiográficos de 854 cães atendidos no HV-UFMG, no período de maio de 2001 a dezembro de 2006. Os animais foram encaminhados por médicos veterinários do HV-UFMG e de clínicas particulares da cidade de Belo Horizonte. No levantamento dos dados,

Recebido em 21 de junho de 2008

Aceito em 31 de agosto de 2009

E-mail: michellegc@ig.com.br consideraram-se: raça, idade, sexo e presença de alterações ecocardiográficas.

Todos os exames foram realizados por um mesmo examinador, utilizando um aparelho de ultrassonografia $^{1}$ composto pelos modos $\mathrm{M}$, bidimensional e Doppler (pulsado, contínuo e por mapeamento em cores). A metodologia dos exames e os valores normais de referência utilizados seguiram os preconizados pela literatura (Thomas et al., 1994; Boon, 1998).

Dos 854 animais avaliados, 464 cães eram machos $(54,3 \%)$ e 390 eram fêmeas $(45,7 \%)$, com idades variando de 2 meses a 19 anos. As principais raças encontradas foram Poodle $(n=289)$, Cocker Spaniel Inglês $(n=84)$, Yorkshire Terrier $(n=67)$, Pinscher Miniatura $(n=64)$, Dachshund $(n=43)$, Boxer $(n=26)$, Terrier Brasileiro $(n=21)$, Pastor Alemão $(n=18)$, Schnauzer Miniatura $(\mathrm{n}=17)$, Bichon Frisé $(\mathrm{n}=13)$, Labrador Retriever $(\mathrm{n}=12)$, Basset Hound $(n=9)$, Pequinês $(n=8)$, Shi Tzu $(n=8)$, Beagle $(n=7)$, Spitz Alemão $(n=7)$, Fila Brasileiro $(n=5)$, sem raça definida $(n=102)$ e outras raças $(n=54)$.

Dos 854 cães estudados, $750 \quad(87,8 \%)$ apresentaram alguma alteração cardíaca no exame ecodopplercardiográfico (Tab.1), enquanto $104(12,2 \%)$ foram considerados normais quanto à avaliação cardíaca. Destes, 74 foram encaminhados por apresentarem um ou mais dos seguintes sinais clínicos: tosse, dispneia, e cianose ou síncope. É importante mencionar que a raça Yorkshire Terrier foi a

${ }^{1}$ Ecocardiógrafo HP Sonos 100 CF - Hewlett Packard - EUA. 
mais frequente dentre os animais considerados normais no exame ecodopplercardiográfico, demonstrando que a sintomatologia clínica apresentada possivelmente está relacionada a alguma afecção respiratória, e não cardíaca, podendo explicar o grande número de animais dessa raça submetidos à ecocardiografia. De acordo com Ettinger (1991) e Hawkins (1997), a ecocardiografia é uma ferramenta importante no diagnóstico diferencial de afecções respiratórias e cardíacas, por apresentarem alguns sinais clínicos em comum. Os outros 30 cães sem alterações ecocardiográficas foram levados ao setor para realização de exames pré-cirúrgicos $(\mathrm{n}=10)$ e pré-quimioterapia $(\mathrm{n}=20)$, aplicações emergentes da ecocardiografia.

Tabela 1. Frequência das alterações cardíacas observadas em 750 cães no exame ecodopplercardiográfico, maio de 2001 a dezembro de 2006

\begin{tabular}{lcc}
\hline \multicolumn{1}{c}{ Diagnóstico } & Número de animais & $\%$ \\
\hline Doença valvular adquirida & 575 & 76,7 \\
Cardiomiopatia dilatada & 73 & 9,8 \\
Alterações congênitas & 51 & 6,8 \\
Hipertrofia do ventrículo esquerdo & 24 & 3,2 \\
Hipertensão pulmonar* & 13 & 1,7 \\
Neoplasia & 10 & 1,3 \\
Efusão pericárdica* & 4 & 0,5 \\
Total & 750 & 100 \\
\end{tabular}

*não secundárias a outras patologias cardíacas.

A doença valvular adquirida foi a alteração de maior ocorrência entre as cardiopatias nos cães. Neste grupo, foram incluídos os animais com insuficiência valvular secundária à degeneração mixomatosa ou de causa indeterminada, como endocardite infecciosa.

Mais ainda, $548(95,5 \%)$ pertenciam a raças de pequeno e médio porte, e apenas $26(4,5 \%)$ eram de raças grandes e gigantes, sendo que a raça Poodle a mais acometida. De acordo com estudos anteriores, os machos são mais acometidos por doenças valvulares que as fêmeas (O'Grady et al., 1995; Larsson et al., 2000; Cordeiro e Martin, 2002). Neste levantamento, o número de machos afetados, $326(56,7 \%)$, foi mais alto que o número de fêmeas, 249 (43,3\%), porém não se observou diferença estatística entre sexos.

A degeneração mixomatosa bilateral das válvulas atrioventriculares foi encontrada em 357 (62,1\%) cães, enquanto 198 (34,4\%) mostravam lesão apenas na válvula mitral, e 20 (3,5\%) somente na tricúspide. Segundo O’Grady (1995), a insuficiência da válvula tricúspide, em muitos animais, pode ser secundária ao aumento do ventrículo esquerdo à hipertensão pulmonar, devido à insuficiência cardíaca esquerda, o que pode explicar o envolvimento de ambas as válvulas em um grande número de animais. Apenas um cão, da raça Boxer, apresentava alterações ecocardiográficas sugestivas de endocardite infecciosa, forma rara de doença valvular adquirida em cães.

A cardiomiopatia dilatada (CMD) foi a segunda patologia cardíaca mais diagnosticada entre os animais, observada com maior frequência em machos. Dentre os 73 cães diagnosticados com CMD, 38 eram de raças grandes e gigantes, e 35 da raça Cocker Spaniel. Observou-se aumento da prevalência dessa cardiopatia em animais de médio porte, aspecto já descrito por Gooding et al. (1986) e Kittleson et al. (1997). O elevado número de animais dessa raça deve-se, além de outros fatores, à popularidade da raça na região estudada.

Outra alteração do miocárdio observada foi a hipertrofia concêntrica do ventrículo esquerdo. A cardiomiopatia hipertrófica primária é uma condição rara em cães, mas pode-se encontrar hipertrofia concêntrica secundária à hipertensão sistêmica, ao hipertireoidismo, à estenose subaórtica, entre outras (Calvert, 2002; Kienle e Thomas, 2005). Esse padrão de hipertrofia foi observado em 24 cães. Por se tratar de um estudo retrospectivo, não foi possível determinar as causas primárias dessa alteração. De qualquer forma, a ecocardiografia mostrou-se útil na diferenciação dos padrões de aumento cardíaco, 
tais como: dilatação, hipertrofia concêntrica e excêntrica.

As alterações congênitas diagnosticadas foram: defeito de septo ventricular $(\mathrm{n}=16)$, persistência do ducto arterioso $(\mathrm{n}=10)$, estenose pulmonar $(n=9)$, defeito de septo atrial $(n=7)$, estenose subaórtica $(n=5)$, displasia de tricúspide $(n=4)$ e displasia de mitral $(n=1)$. Foram observadas doenças cardíacas congênitas em $6,0 \%$ de todos os animais estudados, prevalência superior às encontradas por Larsson et al. (2000) e Cordeiro e Martin (2002), que foram de 2,0\% e 1,5\%, respectivamente. Entretanto, esses autores realizaram os exames utilizando apenas os modos $\mathrm{M}$ e bidimensional, e, neste trabalho, os modos Doppler pulsado, contínuo e por mapeamento em cores foram também utilizados. O estudo com o modo Doppler, principalmente o Doppler colorido, facilita a identificação de alterações cardíacas discretas, dificilmente detectadas nos modos $\mathrm{M}$ e bidimensional, como é o caso de pequenos defeitos septais (Bonagura e Miller, 1998; Kienle e Thomas, 2005), podendo explicar o grande número de animais com defeito de septo ventricular.

Neste estudo, 10 animais apresentaram massas cardíacas com características neoplásicas. A localização das neoplasias foi variável, sendo encontradas adjacentes ao átrio direito $(n=6)$, na base do coração $(n=3)$ e no interior do átrio esquerdo $(n=1)$. Observou-se ainda, presença de efusão pericárdica, com ou sem tamponamento cardíaco, em oito cães com neoplasia extracardíaca. A efusão pericárdica também foi identificada em nove casos de insuficiência cardíaca congestiva e um de hipoalbuminemia. Para três outros animais foi firmado $o$ diagnóstico presuntivo de efusão pericárdica hemorrágica idiopática por não apresentarem uma causa de base conhecida.

Um último grupo identificado foi o de cães com sinais de hipertensão pulmonar, que não é uma doença primária do coração, mas que determina diversas alterações cardíacas, identificadas com o auxílio do exame ecocardiográfico. Para estimar a pressão pulmonar, utilizou-se o valor do pico de velocidade do refluxo tricúspide na equação de Bernoulli modificada. Cento e quinze animais apresentavam hipertensão pulmonar secundária à insuficiência cardíaca esquerda. Outros 13 cães, com o mesmo diagnóstico, não tiveram a causa primária determinada. A dirofilariose e a doença pulmonar crônica, entre outras, compõem a lista de diagnósticos diferenciais nesses casos (Hawkins, 1997; Kienle e Thomas, 2005).

A ecodopplercardiografia é um exame complementar de uso relativamente recente que possibilita o diagnóstico de várias alterações cardíacas. Dentre as cardiopatias estudadas, a doença valvular adquirida e a cardiomiopatia dilatada foram as de maior prevalência, ocorrendo principalmente em cães machos de pequeno ou médio porte e em raças grandes ou gigantes, respectivamente. Entretanto, animais de médio porte da raça Cocker Spaniel também apresentam elevada prevalência para a cardiomiopatia dilatada.

Palavras-chave: cão, ecocardiografia, cardiopatia

\begin{abstract}
A retrospective study was carried out to evaluate echodopplercardiographic examinations of 854 dogs referred to the service of echocardiography. This work presents the frequency of the main cardiopathies according to breed, gender, and presence of echocardiographic alterations. The valvular acquired disease was the most common cardiopathy (76.7\%), mainly occurring in male dogs of small breeds. The dilated cardiomyopathy was the second more diagnosed cardiovascular pathology (9.8\%), and most frequently noticed in male dogs of large or giant breeds. However, Cocker Spaniel breed also presented a high prevalence for this cardiopathy.
\end{abstract}

Keywords: dog, echocardiography, cardiopathy 


\section{REFERÊNCIAS BIBLIOGRÁFICAS}

BONAGURA, J.D.; MILLER, M.W. Doppler echocardiography II: color doppler imaging. Vet. Clin. N. Am.: Small Anim. Pract., v.28, p.13611387, 1998.

BOON, J.A. Manual of veterinary echocardiography. Baltimore: Willians \& Wilkins, 1998. 478p.

CALVERT, C.A. Cardiomiopatia canina. In: TILLEY, L.P.; GOODWIN, J.K. Manual de cardiologia para cães e gatos. 3.ed. São Paulo: Roca, 2002. p.133-152.

CORDEIRO, F.F.; DE MARTIN, B.W. A ecocardiografia como método de auxílio ao diagnóstico das doenças cardíacas em pequenos animais. Rev. Clin. Vet., n.39, p.22-32, 2002.

ETTINGER, S.J. Ordering diagnostic tests in cardiovascular disease. Vet. Clin. N. Am.: Small Anim. Pract., v.21, p.1081-1102, 1991.

GOODING, J.P.; ROBINSON, W.F.; MEWS, G.C. Echocardiographic characterization of dilatation cardiomyopathy in the English Cocker Spaniel. Am. J. Vet. Res., v.47, p.1978-1983, 1986.

HAWKINS, E.C. Afecções do sistema respiratório inferior. In: ETTINGER, S.J.; FELDMAN, E.C. Tratado de medicina interna veterinária. 4.ed. São Paulo: Manole, 1997. v.1, p.1080-1142.
KIENLE, R.D.; THOMAS, W.P. Ecocardiografia. In: NYLAND, T.G.; MATTOON, J.S. Ultrassom diagnóstico em pequenos animais. 2.ed. São Paulo: Roca, 2005. p.365-438.

KITTLESON, M.D.; KEENE, B.; PION, P.D. et al. Results of the multicenter spaniel trial (MUST): taurine and carnitine-responsive dilated cardiomyopathy in American Cocker Spaniels with decreased plasma taurine concentration. J. Vet. Intern. Med., v.11, p.204211, 1997.

LARSSON, M.H.M.A.L.; BARBUSCI, L.O.D.; SOARES, E.C. et al. Estudo ecocardiográfico das cardiopatias mais frequentemente diagnosticadas em espécimes caninos. Rev. Bras. Cienc. Vet., v.7, p.68, 2000.

MUZZI, R.A.L. Estudo ecodopplercardiográfico da degeneração mixomatosa crônica da valva mitral em cães. 2002. 78f. Tese (Doutorado) Escola de Veterinária, Universidade Federal de Minas Gerais, Belo Horizonte.

O'GRADY, M.R.; BONAGURA, J.D.; POWERS, J.D. et al. Quantitative cross-section echocardiography in the normal dog. Vet. Radiol. Ultrasound, v.27, p.34-39, 1986.

THOMAS, W.P.; GABER, C.E.; JACOBS, G.J. et al. Recommendations for standards in transthoracic two-dimensional echocardiography in the dog and cat. Vet. Radiol. Ultrasound, v.35, p.173-178, 1994. 